\title{
基于纳米金辅助信号生成顺序堆积的毛细管电泳免疫分析研究
}

\author{
张召香* 奕文秀＼cjkstart张超英＼cjkstart刘玉洁 \\ (青岛科技大学化学与分子工程学院 肿瘤标志物传感分析教育部重点实验室 青岛 266042)
}

\begin{abstract}
摘要 短裸甲藻毒素(BTX)是具有极强毒性的生物毒素, 能够通过食物链传递引起人类中毒. 由于该毒素没有光学和 电化学信号, 检测十分困难. 本工作利用纳米金作为载体, 将辣根过氧化物酶(HRP)和毒素抗体同时固定到纳米金表 面, 通过 $\mathrm{HRP}$ 催化 $\mathrm{H}_{2} \mathrm{O}_{2}$ 氧化邻氨基酚(OAP)产生的电化学信号检测样品中的毒素, 增大纳米金表面 $\mathrm{HRP}$ 和抗体的物 质的量比使电化学信号得到极大增强. 免疫反应样品电动进样引入分离毛细管中, 在毛细管入口端进行顺序堆积在线 富集，使检测灵敏度进一步提高. 该方法通过纳米金辅助信号生成和顺序堆积在线富集技术实现了对扇贝样品中 BTX-B 的快速灵敏检测, 线性范围为 $0.1 \sim 120 \mathrm{ng} / \mathrm{mL}$, 检出限为 $26 \mathrm{ng} / \mathrm{L}$, 检出限比常规酶联免疫分析(ELISA)法低 365 倍.
\end{abstract}

关键词 短裸甲藻毒素 B; 毛细管电泳; 纳米金; 顺序堆积; 免疫分析; 电化学检测

\section{Capillary Electrophoresis Immunoassay by Gold Nanoparticles Assisted Signal Generation and Sequential Stacking}

\author{
Zhang, Zhaoxiang* Luan, Wenxiu Zhang, Chaoying Liu, Yujie \\ (Key Laboratory of Sensor Analysis of Tumor Marker, Ministry of Education, College of Chemistry and Molecular \\ Engineering, Qingdao University of Science and Technology, Qingdao 266042, China)
}

\begin{abstract}
Brevetoxins (BTXs) are highly toxic biotoxin and can cause human intoxication through food chain. The detection of brevetoxins is very difficult due to lack of optical and electrochemical (EC) signal. In this work, we developed an ultrasensitive capillary electrophoresis (CE) immunoassay and EC method for the determination of BTX-B by gold nanoparticles (AuNPs) assisted signal generation and sequential stacking concentration. The AuNPs were synthesized by sodium citrate reduction of $\mathrm{HAuCl}_{4}$ in water. The AuNPs were conjugated with horseradish peroxidase (HRP) and antibody (Ab) to immobilize the HRP and Ab on the AuNPs surface with the molar ratio of HRP/Ab of 9/1. The Ab conjugated on the AuNPs surface incubated with limited amount of BTX-B in standard solution or shellfish samples to produce immunocomplex on the basis of the noncompetitive immunoreactions. Before sample injection, a $\mathrm{NaOH}$ plug with $10 \mathrm{~cm}$ height difference for $150 \mathrm{~s}$ was hydrodynamically injected into the separation capillary. After incubation for $40 \mathrm{~min}$ at room temperature, the immune sample was then electrokinetically injected into the capillary at $10 \mathrm{kV}$ for $330 \mathrm{~s}$. The positively charged analytes migrated rapidly into the capillary and were neutralized and stacked at the boundary between sample and $\mathrm{NaOH}$ plug, which led to the first preconcentration. After sample loading, the capillary inlet vial was changed to low-pH buffer solution, and $\mathrm{H}^{+}$in the buffer solution moved rapidly into the capillary toward cathode across the neutral analytes zone. The neutralized analytes were positively charged again and the injected analytes were further condensed. Next, the formed immunocomplex, unbound HRP-Au-Ab probe and the excess HRP were separated by CE and sensitively detected by EC detection. AuNPs were used as carriers of HRP and $\mathrm{Ab}$ in order to carry out EC detection with the EC signals derived from catalytic reaction of the carried $\mathrm{HRP}$ to the $\mathrm{H}_{2} \mathrm{O}_{2} / o$-aminophenol system. Simultaneously, the EC signal was highly amplified by improving the $\mathrm{HRP} / \mathrm{Ab}$ molar ratio on the surface of AuNPs. The proposed method by AuNPs assisted signal generation and on-line sequential concentration realized the sensitive and rapid determination of BTX-B in shellfish samples. In the range between 0.1 and 120 $\mathrm{ng} / \mathrm{mL}$, the assay allowed quantitative determination of BTX-B and the limit of detection (LOD) was $26 \mathrm{ng} / \mathrm{L}$. The LOD was 365-fold lower than ELISA method. The amplified sensitivity was enhanced by high HRP/Ab ratio at AuNPs surface and sequential preconcentration. The proposed method provides a convenient and sensitive analytical approach for the determination of trace BTX in complex samples.
\end{abstract}

Keywords Brevetoxin B; capillary electrophoresis; gold nanoparticle; sequential stacking; immunoassay; electrochemical detection

\footnotetext{
* E-mail: qustzhzhx@126.com

Received November 12, 2016; published January 10, 2017.

Supporting information for this article is available free of charge via the Internet at http:/sioc-journal.cn.

Project supported by the National Natural Science Foundation of China (No. 21105051).

项目受国家自然科学基金(No. 21105051)资助.
} 


\section{1 引言}

短裸甲藻毒素(BTX) 是一类具有独特结构及药理活 性的海生毒素, 在动物体内浓度低至 $\mathrm{nmol} / \mathrm{L}$ 或 $\mathrm{pmol} / \mathrm{L}$ 范围便呈现活性, 人类食用含 BTX 的贝类严重损害身 体健康, 而且, 食物中这类毒素无法被烹饪去除. 因此, 研究一种快速、灵敏的检测 BTX 的方法具有重要意义. 然而, 该毒素由于没有光学和电化学信号, 检测非常困 难. 传统的检测方法主要有小鼠生物分析法 ${ }^{[1]}$, 高效液 相色谱法 ${ }^{[2-5]}$ 和免疫分析法 ${ }^{[6,7]}$.

毛细管电泳(capillary electrophoresis, CE)免疫分析 (immunoassay, IA)结合了 $\mathrm{CE}$ 的高分离能力和 IA 的高亲 和特异性, 同时具有样品消耗少、分析速度快等优点而 广泛用于蛋白质 ${ }^{[8,9]} 、 \mathrm{DNA}^{[10]}$ 、赤潮毒素 ${ }^{[11 ~ 13]}$ 和致病 菌 ${ }^{[14]}$ 等的检测. 随着纳米科技的迅速发展, 纳米粒子的 自身特性逐渐凸显, 由于其易于合成、比表面积大、生 物兼容性好等特点而广泛用于环境 ${ }^{[15,16]}$ 、疾病 ${ }^{[17]}$ 、病 毒 ${ }^{[18]}$ 及 $\mathrm{DNA}^{[19-21]}$ 等分析检测中. 王青等 ${ }^{[22]}$ 报道了一种 基于纳米金颗粒增强信号的表面等离子体共振生物传 感器用于甲氧檗因高灵敏检测的研究, 检测下限低至 $0.07 \mathrm{pmol} / \mathrm{L}$. 郭颖等 ${ }^{[23]}$ 制备了石墨烯量子点功能化的金 纳米粒子作为过氧化物模拟酶用于葡萄糖检测, 检出限 达 $3.0 \times 10^{-7} \mathrm{~mol} \cdot \mathrm{L}^{-1}$. Ambrosi 等 ${ }^{[24]}$ 在免疫分析中用金纳 米粒子 (AuNPs) 作为多酶载体检测生物标志物, 每个 AuNPs 可以吸附多个酶分子从而提高检测信号. Tseng 课题组用 AuNPs 从复杂基体中萃取氨基硫醇 ${ }^{[25]}$ 和三聚 氧胺 ${ }^{[26]}$ 进行 $\mathrm{CE}$ 分离检测, 证明 AuNPs 对多种分子具有 很高的吸附容量. Miao 等 ${ }^{[27]}$ 报道了 AuNPs 和酶断裂介 导联用信号放大分析 $\mathrm{Ag}^{+}$, 检出限达 $470 \mathrm{fmol} / \mathrm{L}$. Zhang 等 ${ }^{[28]}$ 研究了基于 AuNPs 的 $\mathrm{CE}$ 分离电化学 $\mathrm{IA}$ 检测贝类 毒素, AuNPs 不仅能改变分析物的淌度增大分离度, 同 时使检测信号放大 70 倍. 综上所述, AuNPs 具有很高的 吸附容量, 可以对分析物进行富集检测.

BTX 毒素的基本结构是由 10 到 11 个环状结构构成 的大环多醚类物质, 本文选择一种 BTX-B(其结构见支持 信息图 S1)作为模型化合物, 研究了一种检测 BTX 毒素 的新方法. 利用 AuNPs 做载体, 将 HRP 和毒素抗体 $(\mathrm{Ab})$ 同时固定到 AuNPs 表面, BTX-B 抗原 $(\mathrm{Ag})$ 和固定了 HRP 和 $\mathrm{Ab}$ 的 AuNPs 探针进行非竞争免疫反应, 免疫反应样 品电动进样引入分离毛细管中, 在毛细管入口端进行顺 序堆积在线富集. 通过增大纳米金表面 HRP/Ab 的物质 的量比以及顺序堆积富集双重放大技术, 使检测信号得 到极大提高. 用该方法测定了扇贝样品中的 BTX-B, 证 明该方法在实际样品检测中具有广阔的应用前景.

\section{2 结果与讨论}

\subsection{HRP-AuNPs-Ab 探针}

BTX-B 本身没有 EC 信号, 检测非常困难, 利用
AuNPs 作载体，将 $\mathrm{HRP}$ 和 $\mathrm{Ab}$ 同时固定到 AuNPs 表面, 免疫反应样品进入反应毛细管后，固定在 AuNPs 表面的 $\mathrm{HRP}$ 催化 $\mathrm{H}_{2} \mathrm{O}_{2}$ 氧化 OAP 产生 EC 信号进行检测 ${ }^{[11 ~ 14]}$, 检测电流的大小与 HRP 的量成正比, 从而对样品进行 定量分析(EC 检测原理及检测条件的优化见支持信息 $\mathrm{S} 1$ 电化学检测部分). 图 1 为 AuNPs、HRP-Au-Ab 探针、 $\mathrm{HRP}$ 和 $\mathrm{Ab}$ 的紫外可见谱图, 从图中可以看出, HRP-Au$\mathrm{Ab}$ 探针的紫外吸收曲线中, 在 $280 \mathrm{~nm}$ 和 $400 \mathrm{~nm}$ 出现了 吸收峰, 分别是 $\mathrm{Ab}$ 和 HRP 的吸收峰, 同裸 AuNPs 的谱 峰相比, 形成 HRP-Au-Ab 探针后由于 AuNPs 表面被 $\mathrm{HRP}$ 和 $\mathrm{Ab}$ 覆盖导致 AuNPs 在 $520 \mathrm{~nm}$ 处的吸收降低, 这 说明 $\mathrm{HRP}$ 和 $\mathrm{Ab}$ 成功固定到 AuNPs 表面. 图 2 为 AuNPs、HRP-Au-Ab 探针、 HRP 和 $\mathrm{Ab}$ 的 CE-UV 检测 谱图, 从谱线 $\mathrm{a} \sim \mathrm{d}$ 可以看出，当没有加入 $\mathrm{HRP}$ 和 $\mathrm{Ab}$ 时, 单纯 AuNPs 的 CE 峰重现性不好(谱线 a), 随着 HRP 和 $\mathrm{Ab}$ 的加入, 不重现的 AuNPs 峰减小, 复合物 HRP-Au$A b$ 的峰开始出现(谱线 $b$ ), 当 HRP 和 $A b$ 过量时, AuNPs 表面达到饱和, 出现过量的 HRP 和 $\mathrm{Ab}$ 峰, 同时 HRP-Au-Ab 的峰重现性较好, 峰位置在 AuNPs 峰之前、 $\mathrm{HRP}$ 和 $\mathrm{Ab}$ 峰之后(谱线 $\mathrm{c} \sim \mathrm{d}$ ). 说明在 AuNPs 表面固定 上 $\mathrm{HRP}$ 和 $\mathrm{Ab}$ 可以提高 AuNPs 的稳定性, 通过峰形和峰 位置进一步确定 HRP 和 $\mathrm{Ab}$ 已固定到 AuNPs 表面.

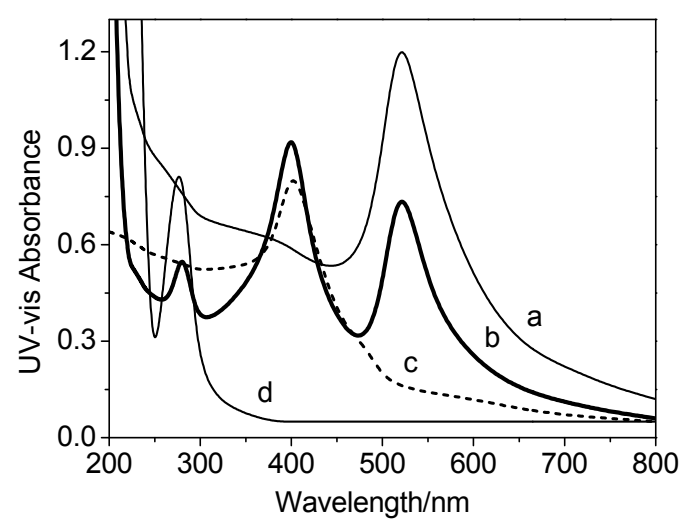

图 1 AuNPs (a), HRP-Au-Ab 探针(b), HRP (c)和 Ab (d)的紫外可见谱 图

Figure 1 UV-vis absorption spectra of AuNPs (a), HRP-Au-Ab probe (b), HRP (c) and Ab (d)

AuNPs 表面积大, 每个 AuNP 能够结合多个生物分 子, 而 EC 检测时, 检测电流的大小与固定在 AuNPs 表 面的 HRP 的量成正比, 因此增大 AuNPs 表面 HRP/Ab 的物质的量比可以提高 $\mathrm{EC}$ 检测的灵敏度. 测定了 AuNPs 表面 HRP/Ab 的物质的量比, 首先测定 AuNPs 吸附的 HRP 和 $\mathrm{Ab}$ 的总量 ${ }^{[29]}$ : HRP、 $A b$ 和 AuNPs 混合 孵育生成 HRP-AuNPs-Ab 探针后, 离心, HRP-AuNPs$\mathrm{Ab}$ 沉淀, 用紫外可见吸收法测出上清液中剩余 HRP 和 $\mathrm{Ab}$ 的量, 孵育前溶液中 $\mathrm{HRP}$ 和 $\mathrm{Ab}$ 的量与孵育后上清 液中 $\mathrm{HRP}$ 和 $\mathrm{Ab}$ 的量之差即为吸附到 AuNPs 上的 HRP 


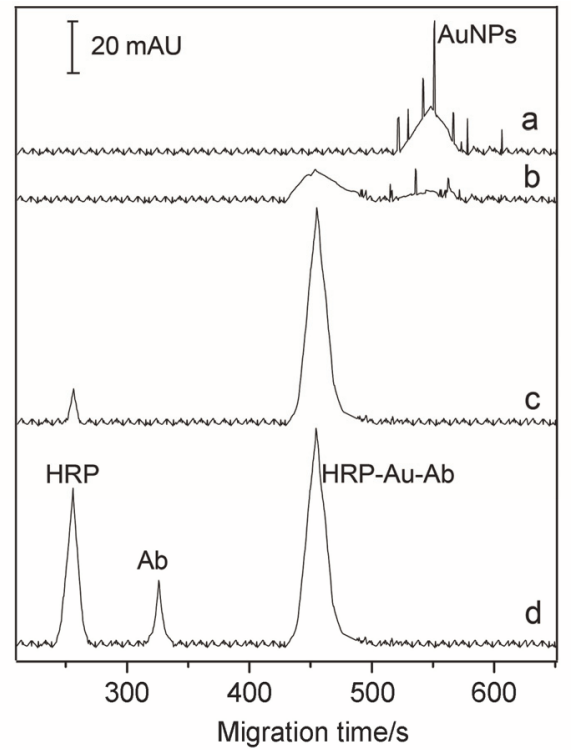

图 2 AuNPs、HRP-Au-Ab 探针、HRP 和 $\mathrm{Ab}$ 的 CE-UV 检测谱图 Figure 2 Electropherograms of AuNPs, HRP-Au-Ab probe, HRP and $\mathrm{Ab}$ by UV detection

a, $C_{\mathrm{HRP}}=0, C_{\mathrm{Ab}}=0 ; \mathrm{b}, C_{\mathrm{HRP}}=5 \mathrm{mg} / \mathrm{L}, C_{\mathrm{Ab}}=2 \mathrm{mg} / \mathrm{L} ; \mathrm{c}, C_{\mathrm{HRP}}=10 \mathrm{mg} / \mathrm{L}, C_{\mathrm{Ab}}$ $=4 \mathrm{mg} / \mathrm{L} ; \mathrm{d}, C_{\mathrm{HRP}}=20 \mathrm{mg} / \mathrm{L}, C_{\mathrm{Ab}}=8 \mathrm{mg} / \mathrm{L}$

和 $A b$, 实验测得 HRP-AuNPs-Ab 探针上吸附的 HRP 和 $\mathrm{Ab}$ 的量为 $14.38 \mu \mathrm{g}$. 测定 HRP-AuNPs-Ab 探针上吸附的 HRP 的量: 用一系列不同浓度的 HRP 溶液分别催化 $\mathrm{OAP}$ 和 $\mathrm{H}_{2} \mathrm{O}_{2}$ 反应进行检测, 绘制标准曲线; 将上述离 心分离的 HRP-AuNPs-Ab 探针分散到溶液中, 在相同条 件下催化 OAP 和 $\mathrm{H}_{2} \mathrm{O}_{2}$ 的反应, 利用标准曲线法计算出 HRP 的量; 吸附在 HRP-AuNPs-Ab 探针上的 HRP 为 $9.97 \mu \mathrm{g}$. 因此, 吸附在 HRP-AuNPs- $A b$ 探针上的 $\mathrm{Ab}$ 为 $4.41 \mu \mathrm{g}$. 吸附在 HRP-AuNPs-Ab 探针上的 HRP/Ab 的物 质的量比为 $9 / 1, \mathrm{HRP}$ 的量是 $\mathrm{Ab}$ 的 9 倍, 可以极大提 高 $\mathrm{EC}$ 检测灵敏度.

按照上述测定方法, 改变 $\mathrm{HRP} / \mathrm{Ab}$ 的物质的量比 $(1: 1 \sim 12: 1)$, 研究了不同物质的量配比对检测灵敏 度和重现性(相对标准偏差, RSD)的影响. 如图 S6(支持 信息)所示, 随着 $\mathrm{HRP} / \mathrm{Ab}$ 物质的量比的增大, 免疫复 合物的峰电流增大, 但当物质的量比大于 $9: 1$ 时, 重现 性变差, 所以选择 $\mathrm{HRP} / \mathrm{Ab}$ 的物质的量比为 $9: 1$.

\section{2 免疫复合物 HRP-AuNPs-Ab-Ag}

HRP-AuNPs-Ab 探针与抗原反应形成免疫复合物, 孵育反应时间明显影响免疫复合物的形成(图 S7, 支持 信息), 随着孵育反应时间的延长, 免疫复合物的峰面 积增大, 当孵育时间为 $40 \mathrm{~min}$ 时, 峰面积达最大值, 继 续增大孵育时间, 复合物的峰面积基本不变, 因此选择 孵育反应时间为 $40 \mathrm{~min}$.

\section{$2.3 \mathrm{CE}$ 分离条件}

$\mathrm{HRP}$ 的最佳催化活性在 $\mathrm{pH} 4$ 左右 ${ }^{[30]}$, 因此 $\mathrm{CE}$ 分离 缓冲液应在酸性范围内, 同时, HRP 是小分子蛋白质,
等电点为 6.5 , 当 $\mathrm{pH}$ 低于 6.5 时, $\mathrm{HRP}$ 带正电荷, 易吸附 在毛细管内壁, 因此在缓冲溶液中加入 PVP 进行动态 涂层. 采用正交试验设计对影响样品分离的主要因素 $\mathrm{BR}$ 缓冲液浓度、 $\mathrm{pH}$ 值和 PVP 浓度对分离条件进行优 化, 如表 1 所示. 根据免疫复合物的峰形、分离效率和 灵敏度, 选择最佳分离缓冲液为 $10.0 \mathrm{mmol} / \mathrm{L} \mathrm{BR}$ 和 $1.0 \%(w / V)$ PVP, $\mathrm{pH} 4.0$.

\section{表 1 正交试验设计 $\mathrm{CE}$ 分离缓冲溶液}

Table 1 Orthogonal experimental design the factors of CE separation buffer

\begin{tabular}{cccc}
\hline No. & $\begin{array}{c}\text { BR concentration/ } \\
\left(\mathrm{mmol} \cdot \mathrm{L}^{-1}\right)\end{array}$ & $\mathrm{pH}$ & PVP concentration/\% \\
\hline 1 & 5.0 & 3.0 & 0.6 \\
2 & 10.0 & 3.0 & 0.8 \\
3 & 15.0 & 3.0 & 1.0 \\
4 & 5.0 & 4.0 & 0.8 \\
5 & 10.0 & 4.0 & 1.0 \\
6 & 15.0 & 4.0 & 0.6 \\
7 & 5.0 & 5.0 & 1.0 \\
8 & 10.0 & 5.0 & 0.6 \\
9 & 15.0 & 5.0 & 0.8 \\
\hline
\end{tabular}

\section{4 顺序富集}

引入一段 $\mathrm{NaOH}$ 区带是该顺序富集法的关键. 高差 $10 \mathrm{~cm}$, 分别引入 $0.1 \mathrm{~mol} / \mathrm{L}$ 的 $\mathrm{NaOH}$ 区带 $90 \mathrm{~s} 、 120 \mathrm{~s}$ 、 $150 \mathrm{~s} 、 180 \mathrm{~s}$ ，改变进样时间发现，随着进样时间增大， 需用碱量增多，如图 3 所示，当进碱时间 $90 \mathrm{~s}$ 时，进样 $160 \mathrm{~s}$ 即获得最佳峰高和分离效率，而进碱时间 $150 \mathrm{~s}$ 时， 进样时间可达 $330 \mathrm{~s}$, 当进碱时间为 $180 \mathrm{~s}$ 时, 由于碱区 带过长, 分离效率降低, 所以选择最佳进碱和进样时间 分别为 $150 \mathrm{~s}$ 和 $330 \mathrm{~s}$.

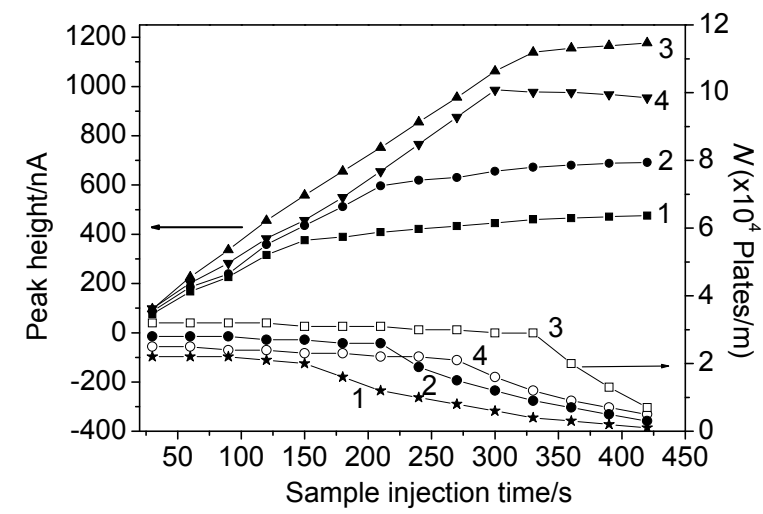

图 3 进样和进碱时间对免疫复合物峰高和分离效率的影响

Figure 3 Effect of sample and base injection time on peak height and separation efficiency

Base injection time: $1,90 \mathrm{~s} ; 2,120 \mathrm{~s} ; 3,150 \mathrm{~s} ; 4,180 \mathrm{~s}$. The running buffer was $10.0 \mathrm{mmol} / \mathrm{L} \mathrm{BR}+1.0 \mathrm{mmol} / \mathrm{L} \mathrm{H}_{2} \mathrm{O}_{2}+1.0 \% \mathrm{PVP}$, $\mathrm{pH} 4.0$

\section{5 基于纳米金辅助信号生成顺序堆积在线富集的 CE 免疫分析 EC 检测 BTX-B}

在最佳条件下检测了加入不同浓度 BTX-B 抗原的 免疫反应样品, 电泳谱图如图 4 所示. 当没有加入毒素 
抗原时, 体系中只有 HRP-AuNPs-Ab 探针和未结合的 $\operatorname{HRP}($ 图 4a); 加入抗原后, 抗原与 AuNPs 探针进行非竞 争免疫反应, 免疫复合物的峰出现(图 4b); 随着抗原浓 度的增大，免疫复合物的峰高增大(图 $4 \mathrm{~b} \sim 4 \mathrm{~d}$ ). 测定了 BTX-B 的浓度 $0.1 \sim 150 \mathrm{ng} / \mathrm{mL}$ 范围内, 免疫复合物的峰 面积与 BTX-B 浓度的关系(图 S8A, 支持信息), 结果表 明, 在 $0.1 \sim 120 \mathrm{ng} / \mathrm{mL}$ 范围内(图 S8B, 支持信息), 免疫 复合物的峰面积与 BTX-B 的浓度成良好的线性关系, 回归方程为 $y=69.32 x+8.76(y$ 为免疫复合物的峰面积, $\mu \mathrm{C} ; x$ 为 BTX-B 的浓度, $\mathrm{ng} / \mathrm{mL})$, 线性相关系数为 0.9982 , 检出限 $(\mathrm{S} / \mathrm{N}=3)$ 为 $26 \mathrm{ng} / \mathrm{L}$.

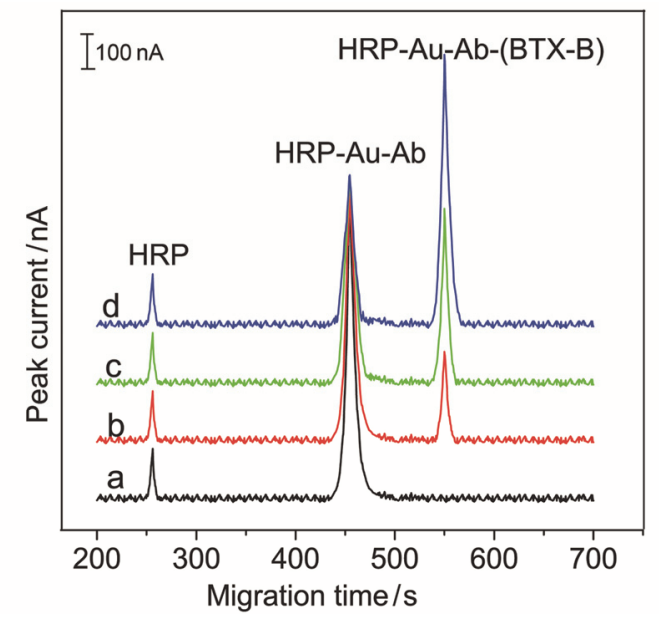

图 4 免疫反应样品的电泳谱图

Figure 4 Electropherograms of immune samples

The concentrations of HRP-Au-Ab probe were constant. Electropherograms of $\mathrm{a}, \mathrm{b}, \mathrm{c}$ and $\mathrm{d}$ correspond to $0,2.0,5.0$, and $10.0 \mathrm{ng} / \mathrm{mL}$ BTX-B, respectively. The running buffer was $10.0 \mathrm{mmol} / \mathrm{L} \mathrm{BR}+1.0 \mathrm{mmol} / \mathrm{L} \mathrm{H}_{2} \mathrm{O}_{2}+1.0 \% \mathrm{PVP}, \mathrm{pH}$ 4.0; OAP concentration: $1.0 \mathrm{mmol} / \mathrm{L}$; Sample injection, $10 \mathrm{kV}$ for $330 \mathrm{~s}$; Separation voltage, $15 \mathrm{kV}$; EC detection potential, $-0.35 \mathrm{~V}$

\section{6 扇贝样品的检测}

为验证方法的实用性和可靠性, 分别用本方法和酶 联免疫吸附分析(ELISA)法测定了 8 组扇贝样品, 如表 2 所示, 两种检测方法的结果一致, 说明本研究方法准确 可靠. 两种分析方法相比, ELISA 法的检出限为 9.5 $\mathrm{ng} / \mathrm{mL}$, 本方法的检出限 $(26 \mathrm{ng} / \mathrm{L})$ 比常规 ELISA 法降低 了 365 倍. 另外, 本法免疫反应在溶液中进行, 反应速 度快, 缩短了分析时间.

本方法是在前期工作 ${ }^{[13]}$ 的基础上进行的，跟前期 工作相比, 本方法不再使用价格昂贵的 BTX 酶标抗原, 实验费用低. 另外, 本方法采用 AuNPs 作载体, 通过增 大吸附在 AuNPs 表面 HRP/Ab 的物质的量比来提高灵 敏度, 检出限比前期工作 ${ }^{[13]}$ 低 4 倍. 用文献 ${ }^{[13]}$ 的方法测 定了 8 种扇贝样品, 结果如表 2 所示, 测定结果与本方 法结果一致, 证明样品中 BTX-B 毒素的含量较低(低于 ELISA 检出限)时, 本方法的测定结果依然具有实用性 和可靠性.
表 2 扇贝样品中短裸甲藻毒素 $\mathrm{B}$ 的检测结果

Table 2 Analytical results of brevetoxin B in shellfish samples $(n=3)$

\begin{tabular}{|c|c|c|c|c|c|c|}
\hline \multirow{3}{*}{$\begin{array}{l}\text { No. of } \\
\text { samples }\end{array}$} & \multicolumn{6}{|c|}{ Content of brevetoxin B in shellfish samples $/\left(\mu \mathrm{g} \cdot \mathrm{kg}^{-1}\right)$} \\
\hline & \multicolumn{2}{|c|}{$\begin{array}{l}\text { The proposed } \\
\text { method }^{a}\end{array}$} & \multicolumn{2}{|c|}{ ELISA $^{b}$} & \multicolumn{2}{|c|}{$\begin{array}{c}\text { The previous } \\
\text { method }^{c}\end{array}$} \\
\hline & Content & $\mathrm{RSD} / \%$ & Content & RSD $/ \%$ & Content & $\mathrm{RSD} / \%$ \\
\hline 1 & 133.2 & 5.7 & 136.5 & 4.4 & 131.6 & 3.8 \\
\hline 2 & 512.6 & 3.5 & 507.8 & 5.9 & 516.7 & 4.4 \\
\hline 3 & 267.5 & 3.9 & 271.2 & 3.6 & 269.9 & 5.2 \\
\hline 4 & 615.2 & 2.2 & 612.6 & 4.8 & 618.7 & 2.6 \\
\hline 5 & 781.9 & 1.6 & 776.3 & 3.2 & 779.6 & 4.9 \\
\hline 6 & 325.5 & 2.8 & 319.1 & 5.1 & 328.3 & 3.3 \\
\hline 7 & 96.2 & 4.5 & 101.7 & 6.3 & 99.7 & 3.7 \\
\hline 8 & 18.7 & 5.3 & $<\mathrm{LOD}$ & - & 21.6 & 5.6 \\
\hline
\end{tabular}

${ }^{a}$ The detection was performed under the optimal conditions of the proposed method. ${ }^{b}$ The detection was performed using the commercial ELISA Check-kit following the manufacturer's procedure. ${ }^{c}$ The detection was performed according to the procedure in reference [13].

为进一步评价方法的准确性, 进行了回收率实验, 在扇贝样品中按低、中、高三个浓度水平 $(0.1,5.0$ 和 50.0 $\mathrm{ng} / \mathrm{mL})$ 添加标准溶液进行回收实验和精密度实验，回收 率在 $93 \% \sim 105 \%$ 之间，相对标准偏差为 $1.2 \% \sim 5.7 \%$, 方法准确可靠，可用于扇贝样品中 BTX-B 的测定.

\section{3 结论}

本工作利用纳米金作为载体，将 $\mathrm{HRP}$ 和 $\mathrm{Ab}$ 同时固 定到纳米金表面，使本没有 EC 信号的 BTX-B 通过 EC 免疫分析得到定量检测; 通过增大 HRP 和 $\mathrm{Ab}$ 的物质的 量比, 使电化学信号得到极大增强, 结合顺序堆积在线 富集技术，使检出限比常规 ELISA 法降低了 365 倍. 该 方法选择性高, 实验耗费低, 在实际样品中 BTX-B 的检 测有良好的应用前景.

\section{4 实验部分}

\section{1 固定 HRP 和 Ab 的 AuNPs 探针及免疫复合物的 制备}

首先合成平均粒径为 $13 \mathrm{~nm}$ 、浓度为 $5.6 \mathrm{nmol} / \mathrm{L}$ 的 AuNPs 溶液 ${ }^{[14,28]}$, 然后以 AuNPs 为载体, 将大量的 HRP 分子和毒素 $\mathrm{Ab}$ 固定到 AuNPs 表面制备 HRP-AuNPs-Ab 探针，具体操作如下：取 $1.0 \mathrm{~mL}$ AuNPs 溶液加入 $0.04 \%$ 的柠檬酸三钠、 $0.02 \%$ 的叠氮化钠和 $0.26 \mathrm{mmol} / \mathrm{L}$ 碳酸钾, 边搅拌边加入 $3.5 \mu \mathrm{L}$ 浓度为 $5.0 \mathrm{mg} / \mathrm{mL}$ 的 HRP 和 $1.5 \mu \mathrm{L}$ 浓度为 $5.0 \mathrm{mg} / \mathrm{mL}$ 的毒素 $\mathrm{Ab}$ (此时 $\mathrm{HRP} / \mathrm{Ab}$ 分子数之比 约为 $9 / 1$ ), 混合均匀后室温孵育 $2 \mathrm{~h}$ 以生成 HRP-AuNPs-Ab(如图 5a).

$$
\mathrm{HRP}+\mathrm{AuNPs}+\mathrm{Ab} \rightarrow \mathrm{HRP}-\mathrm{AuNPs}-\mathrm{Ab}
$$

加入 $100 \mu \mathrm{L} 1 \%$ 的牛血清白蛋白(BSA)终止液, 室 温下搅拌 $30 \mathrm{~min}$, 然后在 $4{ }^{\circ} \mathrm{C}$ 下 $15000 \mathrm{r} / \mathrm{min}$ 离心 20 min, HRP-AuNPs-Ab 结合物用含 $1 \%$ BSA 和 $0.05 \%$ 吐温 20 的 BR 缓冲液洗涤, 最后用 $100 \mu \mathrm{L} 1 \%$ 的 BSA 再悬浮, $4{ }^{\circ} \mathrm{C}$ 下保存备用. 
制备免疫复合物 HRP-AuNPs-Ab-Ag: 在 $200 \mu \mathrm{L}$ 的 微孵育管中加入 $10 \mu \mathrm{L}$ 毒素抗原 $(\mathrm{Ag})$ 标准液或扇贝样品 提取液, 再加入 $10 \mu \mathrm{L}$ 上述制备的 HRP-AuNPs-Ab 探针 溶液, 用 $10 \mathrm{mmol} / \mathrm{L}$ 的 $\mathrm{BR}$ 缓冲液稀释至 $50 \mu \mathrm{L}$, 混合均 匀后室温孵育 $40 \mathrm{~min}$, 毒素抗原与 AuNPs 探针结合的 抗体进行非竞争免疫反应生成免疫复合物 HRP-AuNPs-Ab-Ag(如图 5b), 免疫样品溶液中还有过 量的 AuNPs 探针:

$A g+$ HRP-AuNPs-Ab( 过 量 $) \rightarrow$ HRP-AuNPs-Ab-Ag + HRP-AuNPs-Ab(剩余)

(a)

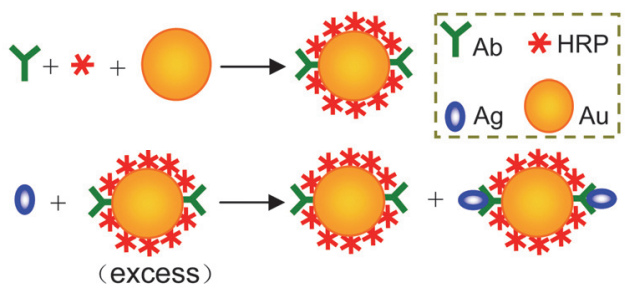

图 5 固定 $\mathrm{HRP}$ 和 $\mathrm{Ab}$ 的 AuNPs 探针(a)及免疫复合物(b)的制备

Figure 5 Preparation of HRP-AuNPs-Ab probes (a) and HRP-AuNPs$\mathrm{Ab}-\mathrm{Ag}$ immune complex (b)

\section{2 基于顺序堆积在线富集的 CE 免疫分析 EC 检测 BTX-B 免疫反应样品}

基于顺序堆积在线富集的 $\mathrm{CE}$ 免疫分析 $\mathrm{EC}$ 检测 BTX-B 免疫样品的原理如图 6 所示. 毛细管内首先充满 运行缓冲液, $10 \mathrm{~cm}$ 高差引入 $\mathrm{NaOH}$ 区带 $150 \mathrm{~s}$ (图 6a). 10 $\mathrm{kV}$ 电动进样, 免疫样品中的正离子快速向毛细管负极 端迁移, 同时 $\mathrm{NaOH}$ 区带中的 $\mathrm{OH}^{-}$向毛细管正极端迁 移, 在毛细管入口端, 样品离子与迎面而来的 $\mathrm{OH}^{-}$发生 中和反应失去正电荷, 堆积在毛细管入口端, 样品离子

(a)

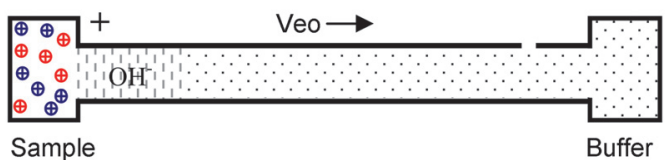

(b)

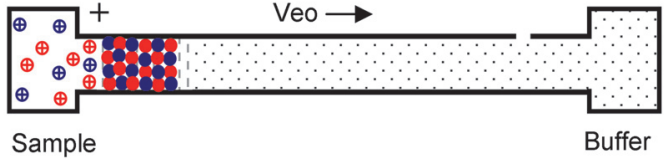

(c)

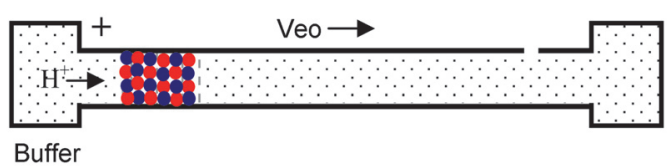

(d)

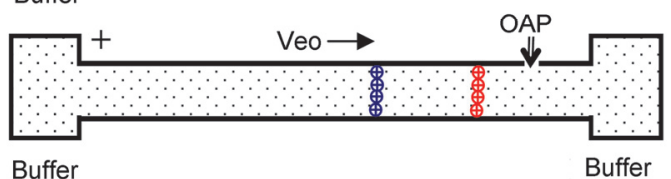

图 6 基于顺序堆积在线富集的 CE 免疫分析 EC 检测 BTX-B 免疫反 应样品

Figure 6 Schematic protocol of CE separation with EC detection of brevetoxin $\mathrm{B}$ immune sample based on sequential stacking preconcentration. (a) introduction of $\mathrm{NaOH}$ plug; (b) base stacking preconcentration; (c) acid stacking concentration; (d) CE separation
进行碱堆积预富集(图 6b). 进样结束后，毛细管入口端 换上缓冲溶液，缓冲液中的 $\mathrm{H}^{+}$快速向阴极迁移，扫过 样品带时使待测样品重新带上正电荷，由于毛细管入口 端的样品首先带上正电荷，迁移速率加快，使样品区带 得到进一步压缩, 堆积在样品与缓冲液的界面上(图 6c). 由于碱-酸顺序堆积压缩样品区带，可使进样时间长达 $330 \mathrm{~s}$ 仍保持较高的分离效率。然后，免疫复合物 HRP-AuNPs-Ab-Ag、AuNPs 探针和过量的 HRP 在分离 毛细管中分成不同的区带(图 6d), 顺次进入反应毛细管 催化 $\mathrm{H}_{2} \mathrm{O}_{2}$ 氧化 OAP 进行 $\mathrm{EC}$ 检测.

\section{References}

[1] Official Methods of Analysis, 14th ed., Association of Official Analytical Chemists, Arlington, VA, 1984, Section 18.086.

[2] Hua, Y.; Lu, W.; Henry, M. S.; Pierce, R. H.; Cole, R. B. Anal. Chem. 1995, 67, 1815.

[3] Nozawa, A.; Tsuji, K.; Ishida, H. Toxicon 2003, 42, 91.

[4] Wang, Z.; Plakas, S. M.; Said, K. R. E.; Jester, E. L. E.; Granade, H. R.; Dickey, R. W. Toxicon 2004, 43, 455.

[5] Wang, Z.; Ramsdell, J. S. Chem. Res. Toxicol. 2011, 24, 54.

[6] Naar, J.; Bourdelais, A.; Tomas, C.; Kubanek, J.; Whitney, P. L.; Flewelling, L. Environ. Health Perspect. 2002, 110, 179.

[7] Bottein, M.-Y. D.; Fuquay, J. M.; Munday, R.; Selwood, A. I.; Ginkel, R. V.; Miles, C. O.; Loader, J. I.; Wilkins, A. L.; Ramsdell, J. S. Toxicon 2010, 55, 497.

[8] Yang, W. C.; Schmerr, M. J.; Jackman, R.; Bodemer, W.; Yeung, E. S. Anal. Chem. 2005, 77, 4489.

[9] Liu, Y.; Mei, L.; Liu, L.; Peng, L.; Chen, Y.; Ren, S. Anal. Chem. 2011, 83, 1137

[10] Wang, X.; Song, Y.; Song, M.; Wang, Z.; Li, T.; Wang, H. Anal. Chem. 2009, 81, 7885 .

[11] Zhang, X. W.; Zhang, Z. X. Toxicon 2012, 59, 626.

[12] Zhang, X. W.; Zhang, Z. X. J. Food Compos. Anal. 2012, $28,61$.

[13] Zhang, X. W.; Zhang, Z. X. J. Chromatogr. Sci. 2013, 51, 107.

[14] Zhang, Z. X.; Zhang, F.; Liu, Y. Acta Chim. Sinica 2012, 70, 2251 (in Chinese). (张召香, 张飞, 刘营, 化学学报, 2012, 70, 2251.)

[15] Liu, X.; Wu, Z.; Zhang, Q.; Zhao, W.; Zong, C.; Gai, H. Anal. Chem. 2016, $88,2119$.

[16] Zhou, G.; Chang, J.; Pu, H.; Shi, K.; Mao, S.; Sui, X.; Ren, R.; Cui, S.; Chen, J. ACS Sens. 2016, 1, 295.

[17] Yang, H.; Fung, S.; Xu, S.; Sutherland, D. P.; Kollmann, T. R.; Liu, M.; Turvey, S. E. ACS Nano 2015, 9, 6774.

[18] Paul, A. M.; Fan, Z.; Sinha, S. S.; Shi, Y.; Le, L.; Bai, F.; Ray, P. C. J. Phys. Chem. C 2015, 119, 23669.

[19] Ma, Z. Y.; Ruan, Y. F.; Xu, F.; Zhao, W. W.; Xu, J. J.; Chen, H. Y. Anal. Chem. 2016, 88, 3864.

[20] Harimech, P. K.; Gerrard, S. R.; El-Sagheer, A. H.; Brown, T.; Kanaras, A. G. J. Am. Chem. Soc. 2015, 137, 9242.

[21] Su, S.; Sun, H.; Cao, W.; Chao, J.; Peng, H.; Zuo, X.; Yuwen, L.; Fan, C.; Wang, L. ACS Appl. Mater. Interfaces 2016, 8, 6826.

[22] Wang, Q.; Zhu, H. Z.; Yang, X. H.; Wang, K. M.; Yang, L. J.; Ding, J. Acta Chim. Sinica 2012, 70, 1483 (in Chinese). (主青, 朱红志, 羊小海, 王柯敏, 杨丽娟, 丁静, 化学学报, 2012, 70, 1483.)

[23] Guo, Y.; Li, W. W.; Zheng, M. Y.; Huang, Y. Acta Chim. Sinica 2014, 72, 713 (in Chinese). (郭颖, 李午戍, 郑敏燕, 黄怡, 化学学 报, 2014, 72, 713.)

[24] Ambrosi, A.; Airò, F.; Merkoci, A. Anal. Chem. 2010, 82, 1151.

[25] Chang, C. W.; Tseng, W. L. Anal. Chem. 2010, 82, 2696.

[26] Chang, C. W.; Chu, S. P.; Tseng, W. L. J. Chromatogr. A 2010, 1217, 7800 .

[27] Miao, P.; Ning, L.; Li, X. Anal. Chem. 2013, 85, 7966.

[28] Zhang, Z. X.; Li, X.; Ge, A.; Zhang, F.; Sun, X.; Li, X. Biosens. Bioelectron. 2013, 41, 452 .

[29] Bradford, M. M. Anal. Biochem. 1976, 72, 248.

[30] Wang, J.; Huang, W.; Liu, Y.; Cheng, J.; Yang, J. Anal. Chem. 2004, 76,5393

(Cheng, B.) 